\title{
Effect of Radiated Field Due to ESD on Ones and Zeroes of the Serial Data Stream
}

\author{
Rajashree Narendra, ${ }^{1, *}$ M. L. Sudheer ${ }^{2}$ \\ ${ }^{1}$ Dept. of TE, BNM IT, Bangalore, 560070, India \\ ${ }^{2}$ Dept. of ECE, UVCE, Bangalore, 560001, India
}

\begin{abstract}
The circuit with an output of specified serial data stream has been exposed to the radiated electromagnetic fields generated by indirect discharge from the ElectroStatic Discharge (ESD) simulator. The circuit malfunctioned at a distance of $35 \mathrm{~cm}$ from the point of discharge. The ESD transient pulse introduced affected only the data stream and binary counter Integrated Circuit (IC) SN74LS393N was found to be malfunctioning. The counter IC passed the parameter test but failed functionally as all the output pins were malfunctioning. The circuit that failed did not have decoupling capacitors connected to the $\mathrm{V}_{\mathrm{cc}}$ supply. Another circu it designed with decoupling capacitors connected to the $\mathrm{V}_{\mathrm{cc}}$ supply of all the IC's has been exposed to indirect ESD air discharge. The effect of the ESD transient pulse on the ones and zeroes of the specified data stream has been observed. The amplitude of the serial data stream has been affected but the order of transmission of the bits has not changed. If the change in amplitude is greater than the set threshold then the order of transmission of bits can change and this becomes critical for sensitive circuits where the set threshold is very small. The decoupling capacitors provided protection against any malfunction of the integrated circuits
\end{abstract}

Keywords Electrostatic discharge, Transient, Electromagnetic Radiated Fields, ESD Simulator, Indirect discharge, Integrated Circu it, Dig ital data

\section{Introduction}

Malfunctioning of integrated circuits and electronic systems have been mathematically predicted for the circuits exposed to the radiated fields $[1,2]$ generated by indirect discharge from an ESD simulator[3]. The way in which an IC fails as a result of ESD varies for every case and it is dependent upon a number of factors including the way in which the charge is dissipated to the topology within the IC. One of the most obvious way in which an IC can fail occurs when the static charge represented as a very high voltage gives rise to a high peak current causing burnout. It is quite possible for the voltage to breakdown an insulating oxide layer leaving the IC permanently damaged as in the case of digital devices [4].

Electronic components are often required to survive a maximum of $8 \mathrm{kV}$ (contact) or $15 \mathrm{kV}$ (air) discharge according to IEC 61000-4-2 ESD standard. Even with suitable ESD protection circuits in the integrated circuits, the IC's are still damaged by the ESD discharge. In many cases the ESD discharge may be greater, so it is wise to provide additional protection[5-13]. The problem of protection becomes more serious in the circuits which have

* Corresponding author:

rajashree.narendra@gmail.com (Rajashree Narendra)

Published online at http://journal.sapub.org/ijea

Copyright (C) 2012 Scientific \& Academic Publishing. All Rights Reserved
IC's not protected with decoupling capacitors at their $\mathrm{V}_{\mathrm{Cc}}$ terminal. It is necessary to keep the high frequency energy from entering the chip, so the capacitors are used for high frequency decoupling. The decoupling capacitors provide a low impedance path to shunt transient energy to ground at the source[14-16].

The ESD indirect discharge test was carried out to verify the ESD immunity of the IC's used in the circuit generating an output of specified data stream. The malfunctioning of the IC occurred and the ESD transient introduced in the data affected the data stream. This happened only in the circuit which had IC's without the external protection offered in the form of decoupling capacitors connected at the supply point of the IC. The circuit which had the external protection of decoupling capacitors connected at the supply point had momentary ESD transient introduced in the data stream, but this did not cause malfunctioning of the IC's or upset the specified data stream. The amplitude of the data is affected which becomes important for the sensitive circuits whose set threshold is very small. So an effective ESD protection solution in the form of decoupling capacitors has been implemented for improving the ESD robustness of the integrated circuits used in the electronic systems.

\section{Testing of Circuit}

A simple circuit as shown in Figure 1 is designed which gives an output of specified data stream. The 8 bit data can 
be changed in the DIP switch provided. We can have a specified stream of one's and zeroes. The data stream chosen is 01110111. The 555 timer is used for clock generation. The clock frequency can be varied from 62.5 $\mathrm{KHz}$ to $118 \mathrm{KHz}$ by varying the $10 \mathrm{~K} \Omega$ potentiometer. The dual 4 stage binary counter IC 74393 is used as an $\div 8$ counter. The IC 74121 is a monostable multivibrator which accepts new data after every 16 bits. The IC 74165 is an 8 bit parallel into serial output shift register. The circuit gives an output of specified serial data stream.

The experimental setup for the indirect test consisted of an ESD simulator Electrometrics EDS 200 which is used to discharge high voltages onto the horizontal ground plane. The test voltage can be varied from $100 \mathrm{~V}$ to $25 \mathrm{KV}$. The very short rise time of $0.7 \mathrm{~ns}$ to $\mathrm{l}$ ns of each single pulse generates a wide RF spectrum and interference. The distance between the ESD discharge point and the circuit under test is varied. The ESD simulator source voltage is set at $13.5 \mathrm{kV}$. An ESD pulse is air discharged on the horizontal ground plane at a distance of $35 \mathrm{~cm}$ from the functioning circuit. The clock and serial data stream is monitored on the LeCroy 6100A, $350 \mathrm{MHz}$ Wave runner oscilloscope.

A short duration transient is introduced in the data as well as the clock stream as shown in the Figure 2, which affected the data transmission. The clock and data voltage level is set to $5 \mathrm{~V}$. The transient introduced in the clock as shown in Figure 3, had a top peak of $+16.85 \mathrm{~V}$ and bottom peak of $-7.25 \mathrm{~V}$ and the data stream as shown in Figure 4, had a top peak of $+7.35 \mathrm{~V}$ and a bottom peak of $-4.6 \mathrm{~V}$. As soon as the ESD transient is introduced in the clock and data, the data shown at the top in Figure 2 changed from 1 to 0 and also the clock shown at the bottom in Figure 2 momentarily changed from 1 to 0 for $2.5 \mu \mathrm{s}$.
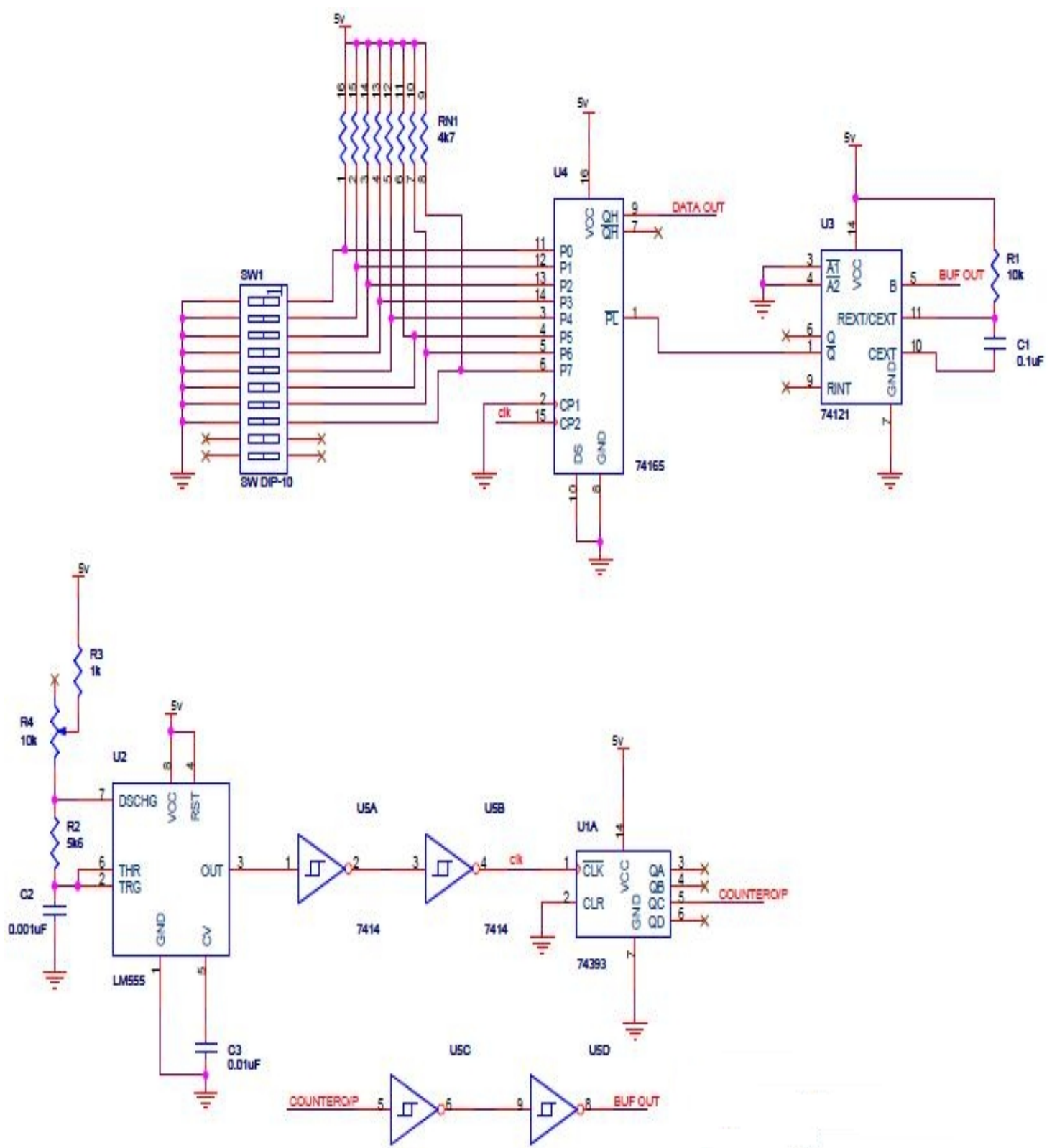

Figure 1. Test Circuit exposed to radiated field from an ESD 


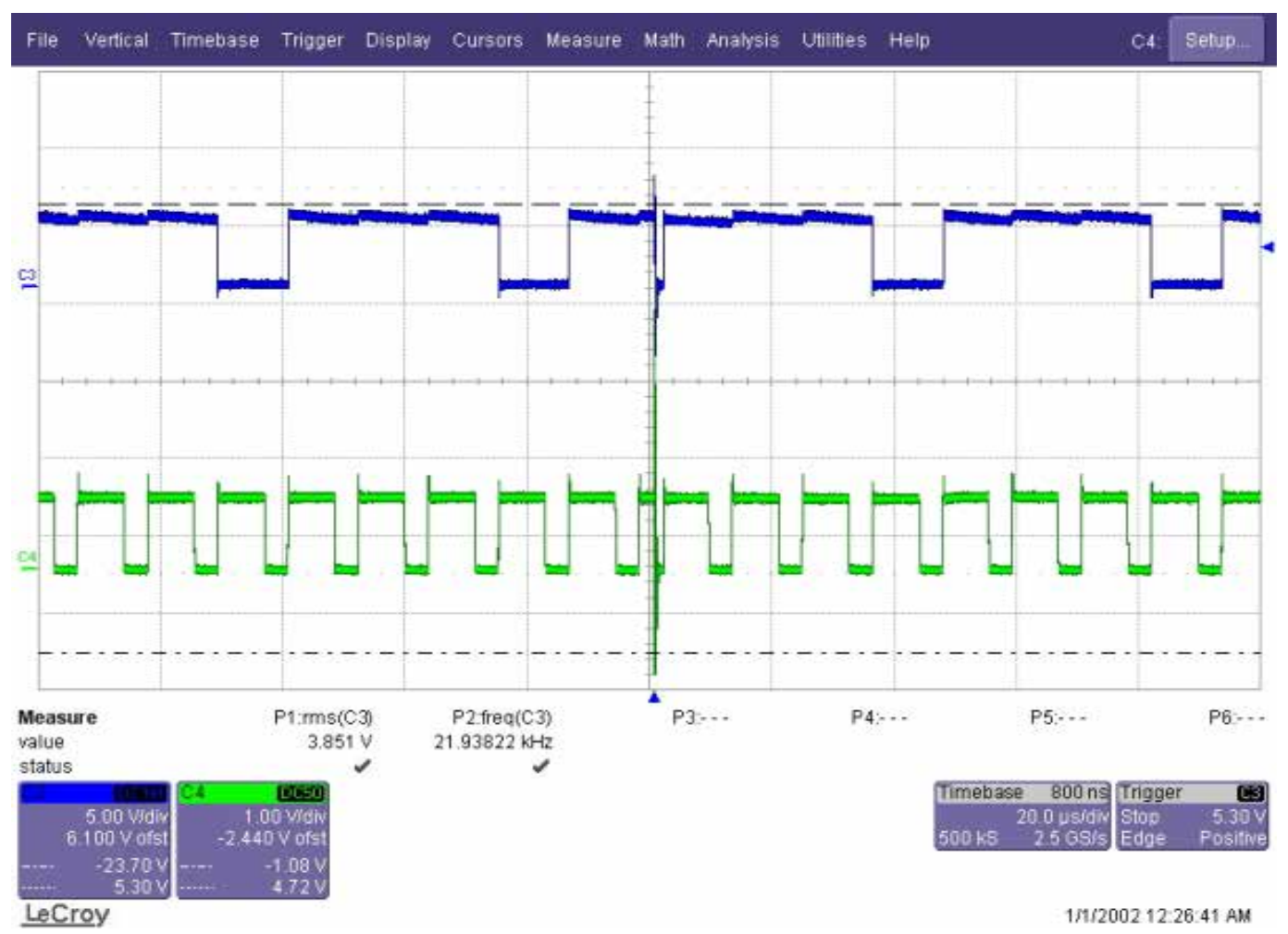

Figure 2. Effect of ESD Transient on the data and clock

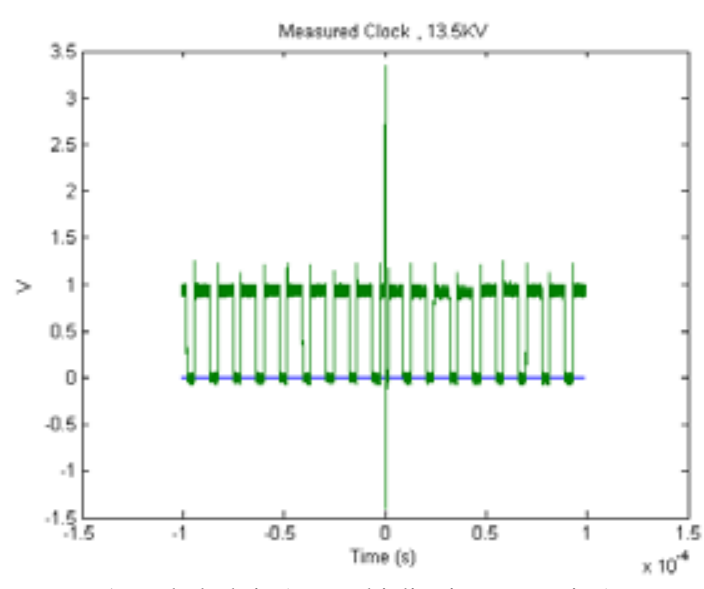

Actual clock is $5 \mathrm{~V}$. Multiplication Factor is 5

Figure 3. Clock stream with the ESD transient

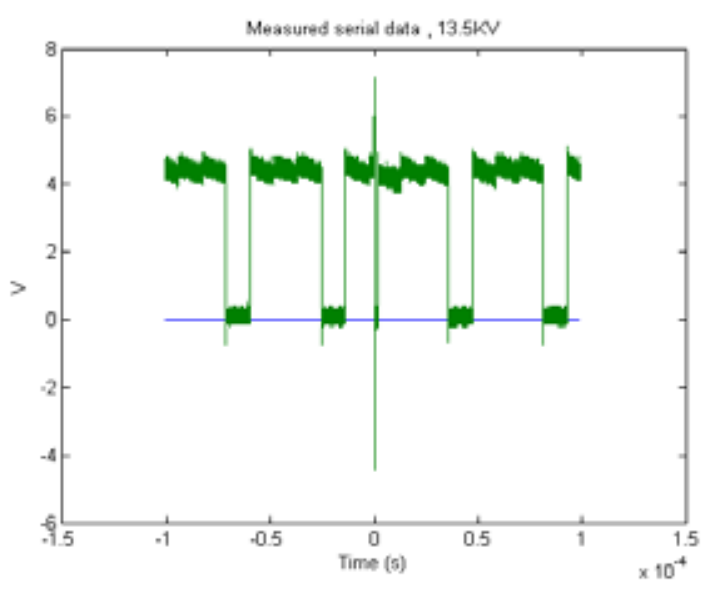

Figure 4. Data stream with the ESD transient

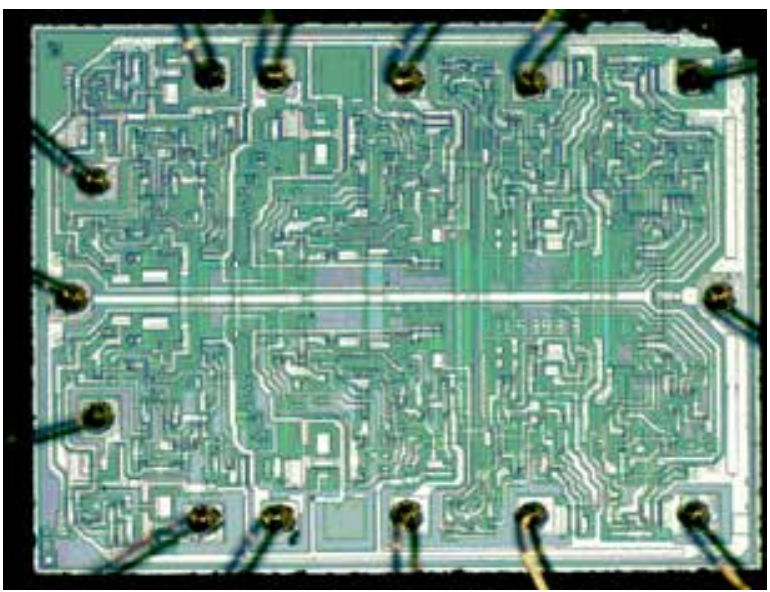

Figure 5. Optical Topography of IC SN74LS393N (Failed device)

\section{Analysis of the ESD Test}

An extra 1 has been added in the data stream 011110111. The malfunctioning of the circuit occurred during the ESD event and the data/clock stream continued for the next 16 bits. But when we tried to change the data, the circuit did not output the specified data but the clock pulse stream gave output as specified. Each point in the circuit is checked and the IC's are also investigated. All the pins of the DIP switch are perfectly working. The Timer IC and Buffer IC are found to be okay. Pin $5(\div 8)$ of the Counter IC SN74LS393N is not giving any output. The IC 74121 monostable multivibrator is not receiving any input at pin 5 (Input B). The data output is thus getting affected at the parallel to serial shift register IC 74165. 
Since all the other ICs are found to be okay, the SN74LS393N binary counter is sent for failure analysis which included the parameter measurement, functional test and decapping of the packaged IC. The SN74LS393N binary counter passed the parameter measurement test but the device failed functionally (all output pins 3, 4, 5, 6, 8, 9, 10, 11 of counter IC are malfunctioning). The optical microscope has been used to find any ESD damaged failure spots in the silicon die. But the optical micrograph shown in Figure 5 does not reveal any ESD damaged failure spots.

\section{Testing of Circuit with ESD Protection}

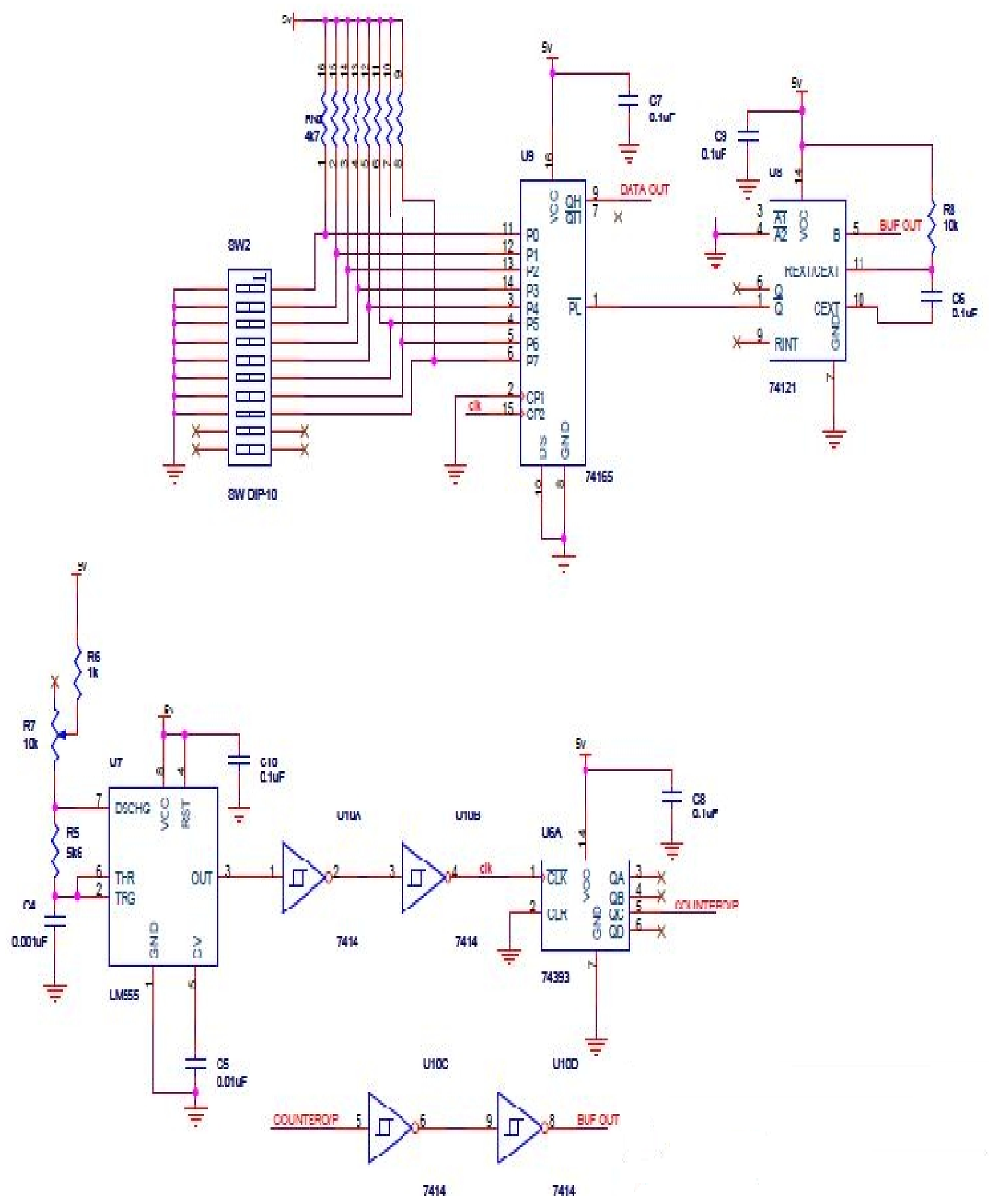

Figure 6. Test Circuit with decoupling capacitors 
The malfunctioning of the counter IC and the change in the data stream is analysed. An effective ESD protection solution in the form of decoupling capacitor is considered for improving the ESD robustness of the IC. Another circu it as shown in Figure 6 is designed with the decoupling capacitors of value $0.1 \mu \mathrm{F}$ connected to the $\mathrm{V}_{\text {cc }}$ supply of all the IC's. This circu it is again exposed to radiated field from an ESD generator. An indirect test on the horizontal coupling plane is done for various voltages and distances. A transient spike of lower magnitude is introduced momentarily but it does not affect the clock stream as well as the specified data stream. So the use of decoupling capacitors reduced the impact of the transient on the functioning of the IC's, which resulted in proper functioning of the circuit. The ESD transient however had an effect on the amplitude of the data stream and its effect on the ones and zeroes of the data stream are observed.

\section{Effect of Radiated Field Due to ESD on Ones of the Data Stream}

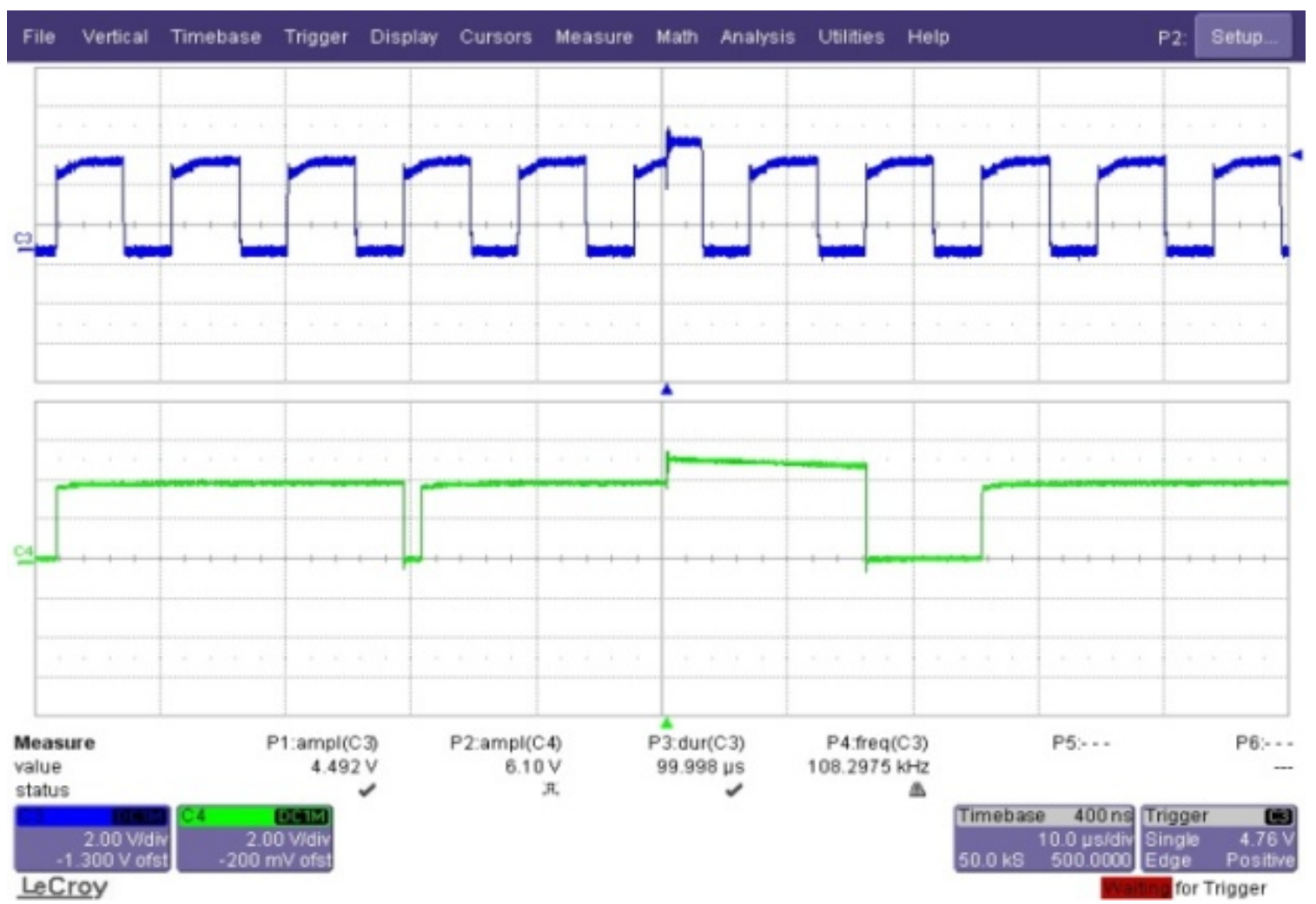

An ESD pulse is air discharged on the horizontal ground plane at various distances $(1 \mathrm{~m}, 0.5 \mathrm{~m}$ and $0.1 \mathrm{~m})$ from the functioning circuit shown in Figure 6. The ESD simulator source voltage is set at $13.5 \mathrm{kV}$. The pulse is discharged during the transmission of Ones of the serial data stream and is monitored on the LeCroy 6100A, $350 \mathrm{MHz}$ Wave runner oscilloscope.

A short duration transient is introduced in the data as well as the clock stream as shown in the Figures 7, 8, and 9, which affects the amplitude of clock and data momentarily but does not affect the order of transmission of clock stream and the data stream[11101111]. In conclusion, it has been observed that when the transient is discharged during the data transmission of ONES, it affects the amplitude of the data but it does not disturb the order of transmission of the bits as the increase in amplitude is well with in the threshold limits.

Another set of data[01010100] is exposed to the indirect ESD test on horizontal plane at $15 \mathrm{kV}$ and at a distance of $0.05 \mathrm{~m}$, the amplitude of the data increased by $2 \mathrm{~V}$ as shown in Figure 10. Since the amplitude increase is within the limits of threshold, the order of transmission of the bits did not change.

Figure 7. Clock and data stream with ESD transient introduced on ONE data bit at the distance of 1 meter 


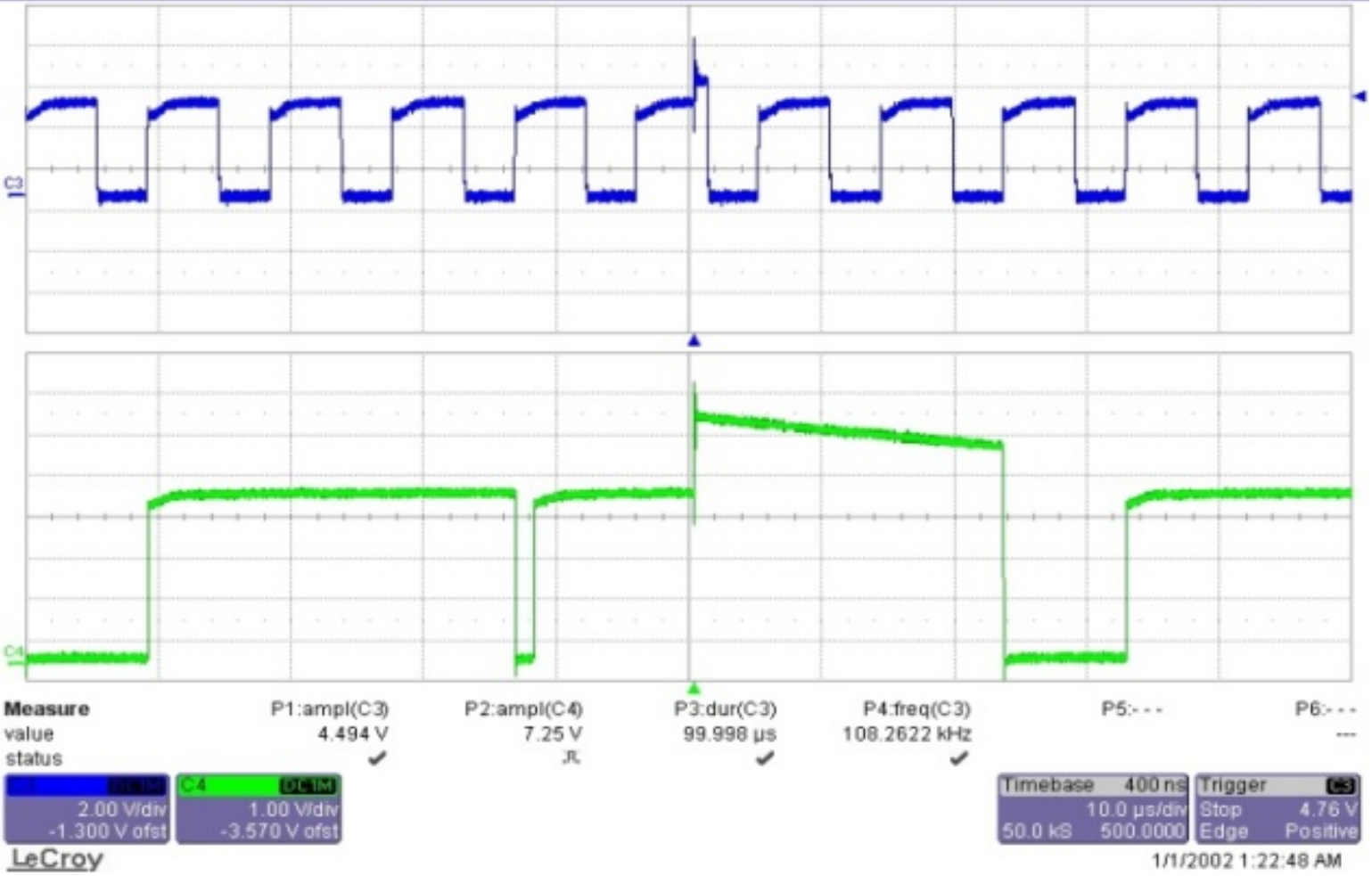

Figure 8. Clock and data stream with ESD transient introduced on ONE data bit at the distance of 0.5 meter

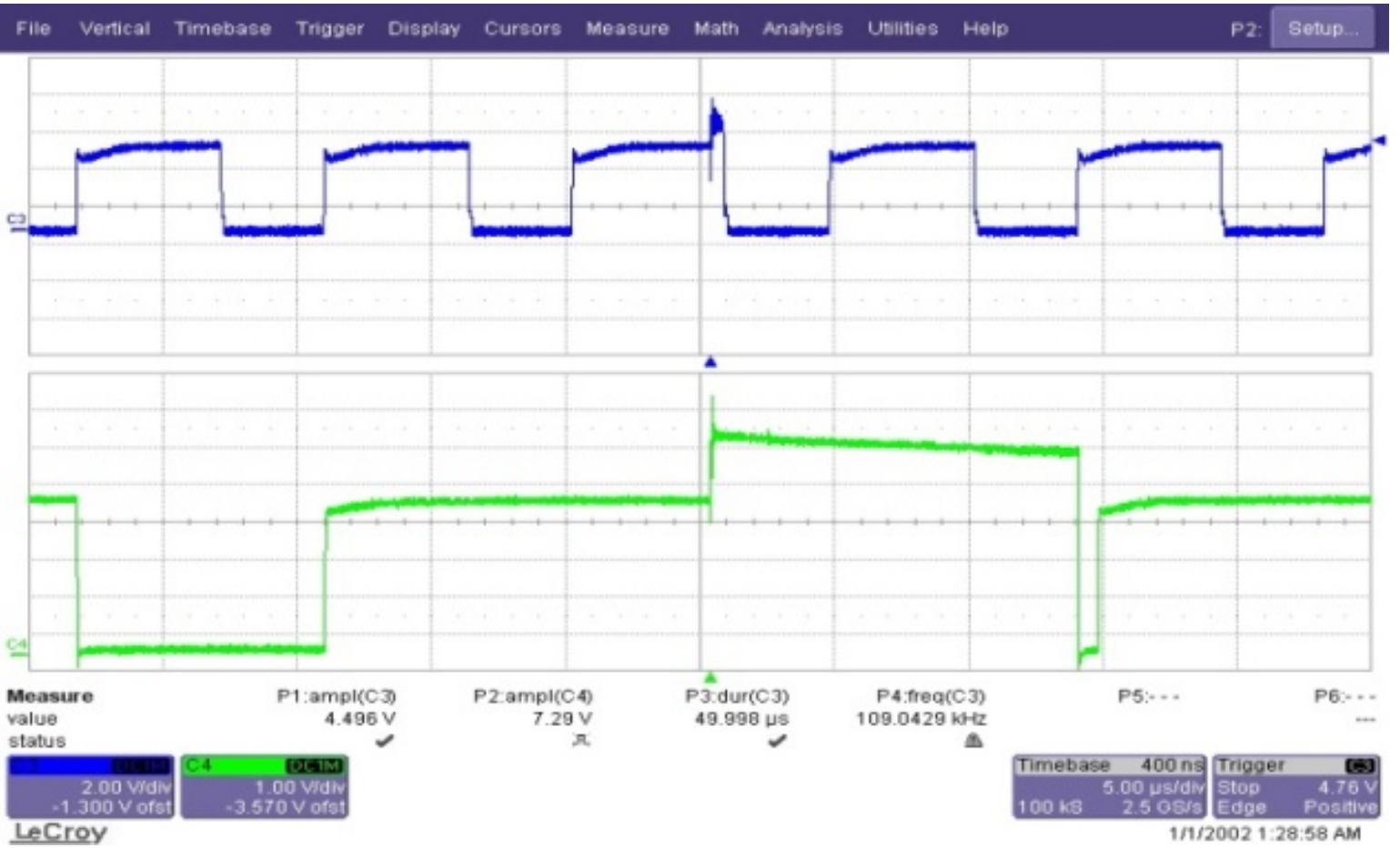

Figure 9. Clock and dat a stream with ESD transient introduced on ONE data bit at the distance of 0.1 meter 


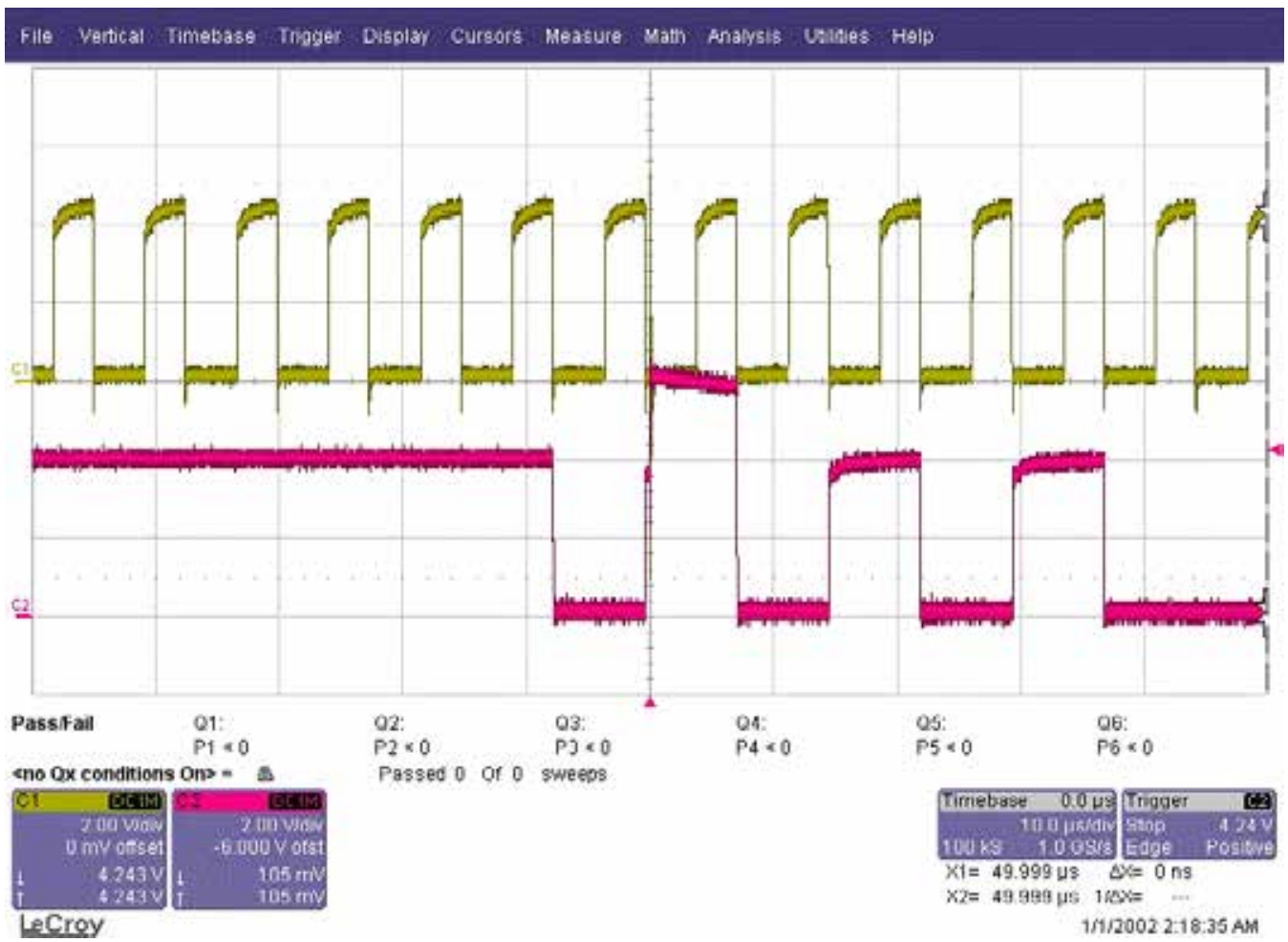

Figure 10. Clock and dat a stream with ESD transient introduced on ONE dat a bit at the distance of $5 \mathrm{~cm}$

\section{Effect of Radiated Field Due to ESD on Zeroes of the Data Stream}

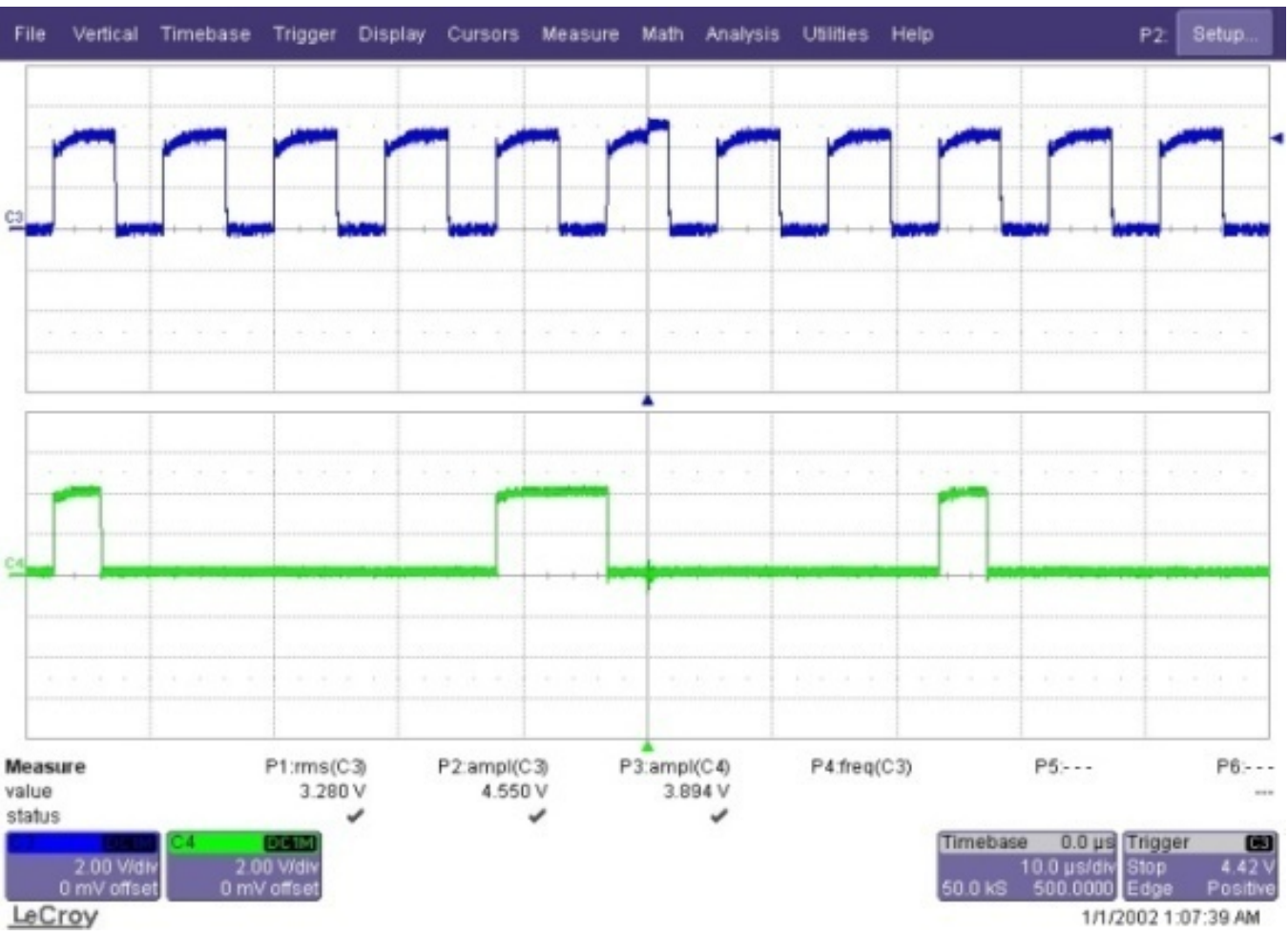

Figure 11. Clock and data stream with ESD transient introduced on ZERO data bit at the distance of 1 met er 


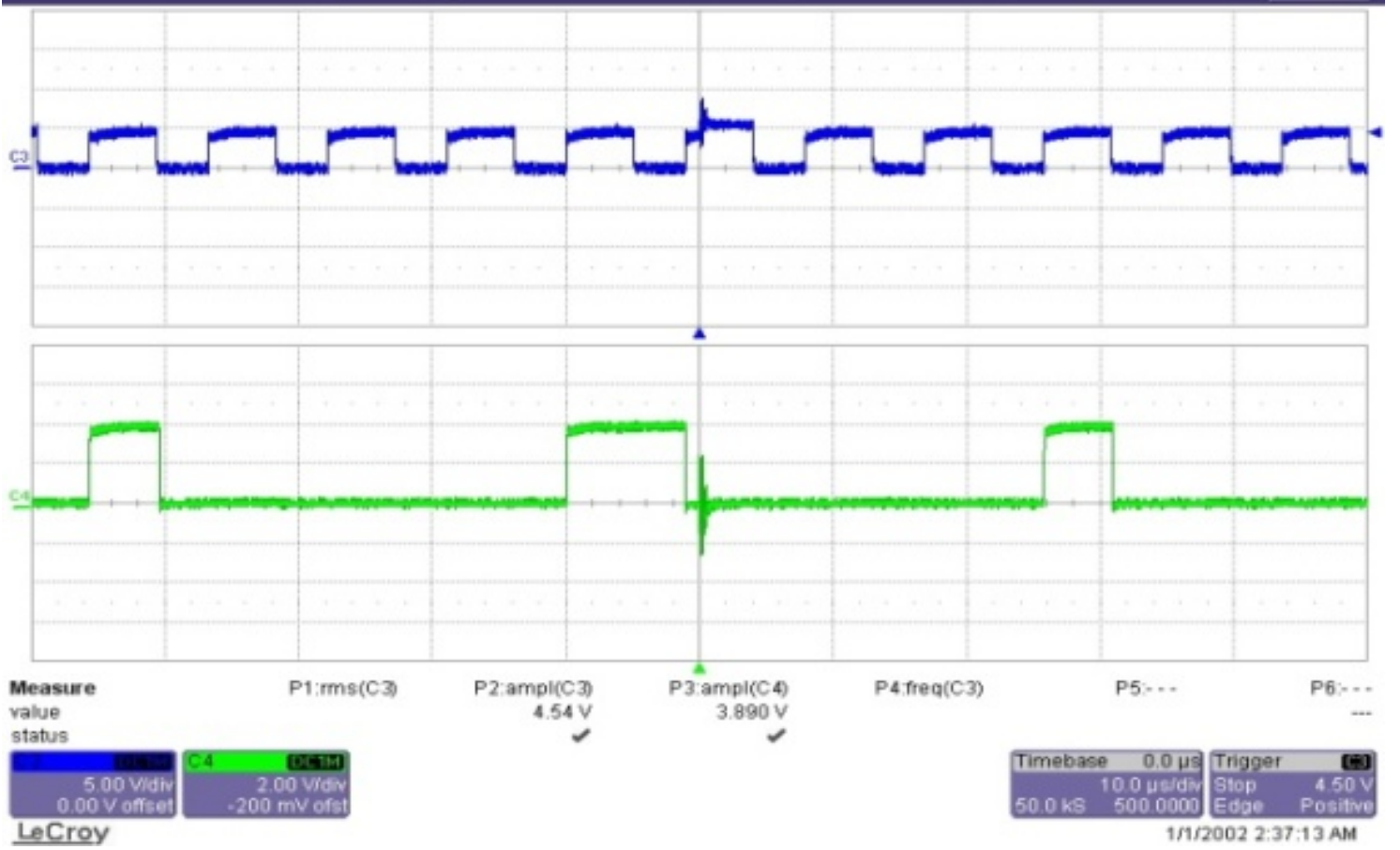

Figure 12. Clock and data stream with ESD transient introduced on ZERO data bit at the distance of 0.5 meter

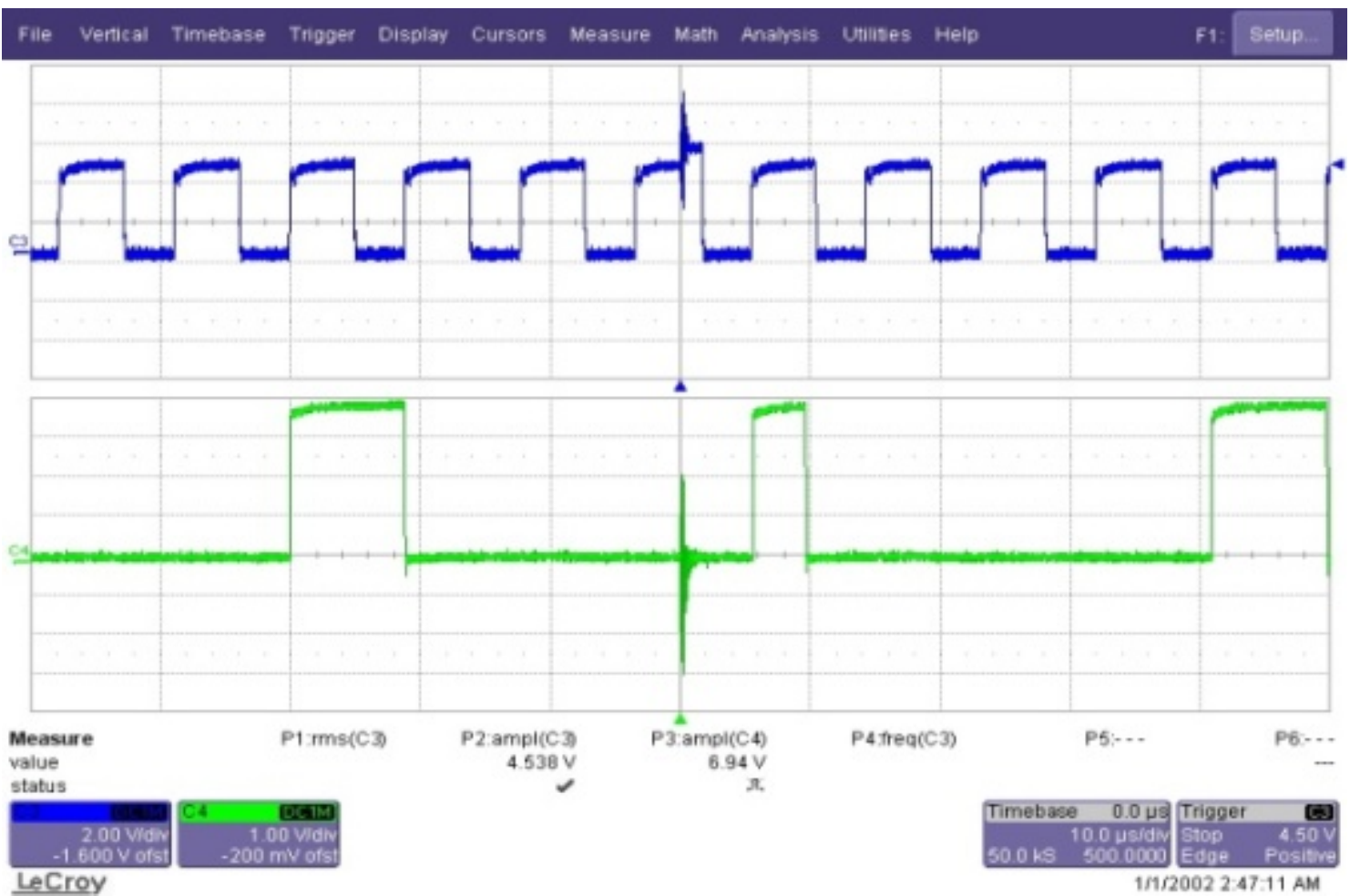

Figure 13. Clock and data stream with ESD transient introduced on ZERO data bit at the distance of 0.1 meter

An ESD pulse is air discharged on the horizontal ground plane at $13.5 \mathrm{kV}$ at various distances $(1 \mathrm{~m}, 0.5 \mathrm{~m}$ and $0.1 \mathrm{~m})$ from the functioning circuit. The ESD pulse is discharged during the transmission of Zeroes of the serial data stream and is monitored.

A short duration transient is introduced in the data as well as the clock stream as shown in the Figures 11, 12 and 13, which affects the amplitude of clock and data momentarily but does not affect the amplitude and the order of transmission of the data stream[00010001]. In conclusion, it is observed that when the transient is discharged during the data transmission of ZEROES, it does not disturb the amplitude of the data and the order of transmission of data bits. 


\section{Conclusions}

The malfunctioning of the IC's due to the effect of the radiated fields from an ESD generator and the effect of ESD transient introduced in the serial data stream is investigated. The results on the malfunctioning of the IC and the circuit failure analysis shows that the IC's need extra external ESD protection. A decoupling capacitor must be connected to the $\mathrm{V}_{\mathrm{cc}}$ supply of each of the individual ICs in the given circuit. By using this proposed effective ESD protection scheme, the ESD robustness of the circuit giving specified serial data stream has been improved. The positive peak of the transient introduced in the data stream has decreased from $+7.35 \mathrm{~V}$ to $+3.08 \mathrm{~V}$. The effect of the ESD transient introduced in the data and clock stream has reduced considerably which resulted in the safe working of the circuit without any malfunction. Also, it has been observed that when the transient is discharged during the data transmission of ONES, it affects the amplitude of the data but it does not disturb the order of transmission of the bits as the increase in amplitude is well within the threshold limits. When the transient is discharged during the data transmission of ZEROES, it does not disturb the amplitude of the data as well as the order of transmission of data bits.

\section{ACKNOWLEDGEMENTS}

Rajashree Narendra would like to thank the EMI/EMC group at LRDE for providing an opportunity to carry out her PhD research work.

\section{REFERENCES}

[1] Rajashree Narendra, M.L.Sudheer, V. Jithesh, D.C. Pande, "Mathematical Analysis of ESD Generated EM Radiated Fields on Electronic Subsystem”, Asia Pacific Symposium on EMC, 2010, pp. 449-452.

[2] S.V.K. Shastry and V.K. Hariharan, "Computer Aided Analysis Of ESD Effects In Dual Gate MOSFET VHF Amplifier”, IEEE International Symposium on EMC, Aug 1990, pp. 424-430.

[3] Robert Ashton, “System level ESD Testing-The Test setup”, Challenges in testing, Conformity, December 2007, pp 34-40.

[4] Ming-Douker, Jeng-Jie Peng and Hsin-Chin Jiang, "Failure Analysis of ESD damage in a high-voltage driver IC and the effective ESD protection solution”, Proceedings of 9th IPFA 2002, Singapore, pp 84-89.

[5] C. Duvvury, R.N.Rountree and O. Adams, “ Internal chip ESD phenomena beyond the protection circuit”, IEEE
Transactions on Electron Devices, Vol. 35, No. 12, 1988, pp 2133-2139.

[6] Hongxia Wanga, Samuel V. Rodrigueza, Cagdas Dirika \& Bruce Jacoba, "Electromagnetic Interference and Digital Circuits: An Initial Study of Clock Networks”, Electromagne tics, Volume 26, Issue 1, 2006, pp 73-86.

[7] H. Wang, J. Li, H. Li, K. Xiao, and H. Chen, "Experimental study and spice simulation of cmos inverters latch-up effects due to high power microwave interference," Progress In Electromagnetics Research, Vol. 87, 2008, pp. 313-330.

[8] Nicolas Lacrampe, Fabrice Caignet, Marise Bafleur, Nicolas Nolhier, Nicolas Mauran, "Characterization and Modeling Methodology for IC's ESD Susceptibility at System Level Using VF-TLP Tester”, EOS/ESD Symposium 2007, 5B-1.1to 5B-1.7.

[9] Tze Wee Chen, Choshu Ito, William Loh, Wei Wang, Kalyan Doddapaneni, Subhasish Mitra, and Robert W. Dutton, "Design Methodology and Protection Strategy for ESD-CDM Robust Digital System Design in 90-nm and 130-nm Technologies, IEEE Transactions on Electron Devices,, Vol. 56, No. 2, February 2009, pp. 275-282.

[10] Shuqing Cao , Akram A. Salman, Stephen G. Beebe, Mario M. Pelella, Jung-Hoon Chun, and Robert W. Dutton, “ESD Device Design Strategy for High Speed I/O in 45nm SOI Technology”, EOS/ESD Symposium 2008, 3A-6.1 to 3A-6.7.

[11] Alexandre Boyer, Amadou Cisse Ndoye, Sonia Ben Dhia, Member, IEEE, Laurent Guillot, and Bertrand Vrignon, "Characterization of the Evolution of IC Emissions After Accelerated Aging”, IEEE Transactions on electromagnetic Compatibility, Vol. 51, No. 4, November 2009, pp. 892-900.

[12] Shuqing (Victor) Cao, Jung-Hoon Chun, Stephen G. Beebe, and Robert W. Dutton, "ESD Design Strategies for High-Speed Digital and RF Circuits in Deeply Scaled Silicon Technologies" IEEE Transactions on Circuits and Systems - I Regular Papers, Vol. 57, No. 9, September 2010, pp. 2301-2311.

[13] Shuqing Cao, Tze Wee Chen, Stephen G. Beebe, Robert W. Dutton, "ESD Design Challenges and Strategies in Deeply scaled Integrated Circuits”, IEEE 2009 Custom Integrated Circuits Conference (CICC), 23-1-1 to 23-1-8.

[14] Zhen Mu and Heiko Dudek, Kun Zhang*, "How to Choose \& Place Decoupling Capacitors to Reduce the Cost of the Electronic Products”, Cadence Design Systems, Inc., * Huawei Technologies Co., Ltd, 2003.

[15] Hubing, Van Doren, Sha, Drewniak, and Wilhelm, “An Experimental Investigation of 4-Layer Printed Circuit Board Decoupling, Proceedings of the 1995 IEEE International Symp osium on Electromagnetic Compatibility, August, 1995, pp. 308-312.

[16] Tamara Schmitz and Mike Wong, "Choosing and Using Bypass Capacitors”, INTERSIL Application Note, AN1325.0, August 3, 2007, pp. 1-10. 